\title{
Actin-dependent activation of serum response factor in T cells by the viral oncoprotein tip
}

Kristin Katsch', Sarah Jill de Jong ${ }^{1}$, Jens-Christian Albrecht ${ }^{1}$, Julia Steger ${ }^{1}$, Harald Genth², Guido Posern ${ }^{3}$ and Brigitte Biesinger ${ }^{1 *}$

\begin{abstract}
Serum response factor (SRF) acts as a multifunctional transcription factor regulated by mutually exclusive interactions with ternary complex factors (TCFs) or myocardin-related transcription factors (MRTFs). Binding of Rhoand actin-regulated MRTF:SRF complexes to target gene promoters requires an SRF-binding site only, whereas MAPK-regulated TCF:SRF complexes in addition rely on flanking sequences present in the serum response element (SRE). Here, we report on the activation of an SRE luciferase reporter by Tip, the viral oncoprotein essentially contributing to human T-cell transformation by Herpesvirus saimiri. SRE activation in Tip-expressing Jurkat T cells could not be attributed to triggering of the MAPK pathway. Therefore, we further analyzed the contribution of MRTF complexes. Indeed, Tip also activated a reporter construct responsive to MRTF:SRF. Activation of this reporter was abrogated by overexpression of a dominant negative mutant of the MRTF-family member MAL. Moreover, enrichment of monomeric actin suppressed the Tip-induced reporter activity. Further upstream, the Rho-family GTPase Rac, was found to be required for MRTF:SRF reporter activation by Tip. Initiation of this pathway was strictly dependent on Tip's ability to interact with Lck and on the activity of this Src-family kinase. Independent of Tip, Tcell stimulation orchestrates Src-family kinase, MAPK and actin pathways to induce SRF. These findings establish actin-regulated transcription in human $T$ cells and suggest its role in viral oncogenesis.
\end{abstract}

Keywords: Actin, Herpesvirus saimiri, Lck, MRTF, Oncoprotein, Serum response factor, T lymphocyte

\section{Background}

Serum response factor (SRF) is widely expressed in both invertebrates and vertebrates. SRF plays an essential role in embryogenesis, but is also involved in multiple processes in developed organisms including neuronal and muscle cell function.

SRF binds as a dimer to a specific DNA sequence known as the CArG box in the promoter of hundreds of target genes. Selective binding is determined by interactions with more than 60 different cofactors, which turn SRF into a versatile transcription factor translating celland stimulus-specific signaling into selective target gene expression [1,2].

Well-known SRF cofactors are members of the ternary complex factor (TCF) family of Ets domain proteins, like Elk-1, SAP-1 and Net. They are regulated by

\footnotetext{
* Correspondence: Brigitte.Biesinger@viro.med.uni-erlangen.de 'Institut für Klinische und Molekulare Virologie, Friedrich-AlexanderUniversität Erlangen-Nürnberg, Erlangen, Germany Full list of author information is available at the end of the article
}

phosphorylation via the classical mitogen-activated protein kinase (MAPK) pathway involving the GTPase Ras, which activates the serine-threonine kinases Raf, MEK and ERK. Their recruitment to DNA depends on a defined DNA sequence, called Ets motif $(\mathrm{C} / \mathrm{A})(\mathrm{C} / \mathrm{A})$ GGA(A/T), next to the SRF-binding CArG box $[3,4]$. A serum response element (SRE), first described in the c-fos promoter, contains an Ets motif adjacent to the CArG box [5].

Another group of SRF cofactors are the myocardinrelated transcription factors (MRTFs). Myocardin, the founding member of this family, is selectively expressed in cardiac and smooth muscle cells and constitutively binds SRF. In contrast, MRTF-A (MAL, MKL1, BSAC) and MRTF-B (MAL16, MKL2) are widely expressed in many cell types [6]. Their cofactor function is controlled by GTPases of the Rho family (RhoGTPases), which are considered as important regulators of the actin cytoskeleton. Activation of the RhoGTPases RhoA, Rac1 and Cdc42 results in the formation of focal adhesion
C Biomed Central 
complexes, lamellipodia and filopodia, respectively [7]. These processes involve actin polymerization and thereby reduce the levels of monomeric, globular actin (G-actin). G-actin binds to N-terminal RPEL motifs of MRTF and thereby sequesters and negatively regulates MRTF. RhoGTPase-mediated reduction of G-actin liberates MRTF, resulting in its nuclear accumulation and SRF cofactor function. SRF-bound MRTF dimers directly contact DNA near the SRF binding sequence. However, a specific MRTF binding sequence, similar to the Ets motif, has not yet been found $[1,6]$.

Differential regulation of SRF target genes is based on gene-specific cofactor preferences and cofactor competition for a common binding site on SRF [8-11]. In this context, specific SRF functions are defined only for a limited set of cell types and assignment of cofactors is lagging. Conditional knock-out approaches were recently used to elucidate the function of SRF and the role of TCFs and MRTFs in mouse T cells. Elimination of SRF by a CD4-Cre transgene at the $\mathrm{CD} 4^{+} \mathrm{CD} 8^{+}$double positive stage impairs $\mathrm{T}$-cell development and results in the absence of peripheral $\mathrm{T}$ cells [12]. An earlier elimination of SRF by a hCD2-Cre transgene at the $\mathrm{CD} 4{ }^{-} \mathrm{CD}^{-}$double negative stage severely reduces the numbers of single positive thymocytes, thymic $\mathrm{T}_{\text {reg }}$ and NK $\mathrm{T}$ cells. Introduction of recombinant SRF lacking the ability to bind TCFs or MRTFs fails to restore thymocyte maturation. In contrast, reconstitution was successful upon introduction of wild-type SRF or a fusion of the recombinant SRF with Elk [13]. While this study documents an essential role of TCF:SRF complexes in T-cell development, activation and function of MRTF:SRF complexes in $\mathrm{T}$ cells remain to be established.

Herpesvirus saimiri (HVS) is the T-lymphotropic prototype of $\gamma 2$-herpesviruses. In contrast to the apathogenic appearance in its natural host, the squirrel monkey (Saimiri sciureus), HVS causes severe T-cell lymphoma in experimentally infected non-natural primate hosts [14]. Most notably, in vitro infection of human peripheral blood mononuclear cells with HVS strain C488 gives rise to continuously proliferating T-cell lines [15]. Deletions of viral genomic sequences coding for the oncoproteins StpC (Saimiri transformation-associated protein of subgroup C) and Tip (Tyrosine kinase interacting protein) obviate human T-cell transformation as well as pathogenicity in non-human primates [16]. Conditional expression of Tip alone in transgenic mice leads to T-cell lymphoma [17]. Tip engages the Src-family kinase (SFK) Lck, a central mediator of proliferation in response to Tcell receptor stimulation $[18,19]$. Lck interaction and activation relies on two motifs in Tip, a sequence homologous to the C-terminus of Src-family kinase domains $(\mathrm{CSKH})$ and a proline-rich Src homology domain 3 binding sequence (SH3B) $[18,20,21]$. The integrity of both motifs, CSKH and SH3B, is required for Tip to support human T-cell transformation [22]. However, pro-proliferative downstream effectors of Tip:Lck interaction are not defined yet. Pro-oncogenic functions are characterized for signal transducer and activator of transcription 3 (STAT3) [23]. Indeed, STAT3 is activated by Lck in the presence of Tip and is constitutively phosphorylated in HVS-C488 transformed lymphocytes [21,24-26]. However, mutation of tyrosine residue 114 (Y114) in Tip abrogates constitutive STAT3 phosphorylation, but not viral transformation of human T cells $[27,28]$. Thus, alternative Tip:Lck effectors must be involved to trigger T-cell proliferation. Given the central role of mitogenactivated protein kinases (MAPK) for growth regulation in general, we previously analyzed MAPK phosphorylation and activation of MAPK-regulated transcription in the presence of the HVS-C488 oncoproteins, StpC and Tip [29]. In Jurkat T cells, neither StpC nor Tip induce the phosphorylation of MEK $1 / 2$ and ERK1/2 or the activity of the MAPK-regulated transcription factor AP-1. Nevertheless, Tip specifically triggers SRF activity in this test system [29].

In this work, we now address the mechanism of SRF activation by the viral oncoprotein Tip. We demonstrate an SRF activation in $\mathrm{T}$ cells that depends on actin polymerization and on the cofactor MAL and is abrogated by dominant-negative Rac1. Tip requires Lck interaction and Src kinase activity to induce this pathway, which may also be a target of $\mathrm{T}$-cell receptor stimulation.

\section{Results}

\section{Tip induces SRF-regulated transcription independent of} MAPK activity

We previously reported activation of a serum response element (SRE) luciferase reporter by the viral oncoprotein Tip in Jurkat T cells. This activation was not accompanied by enhanced ERK1/2 or MEK1/2 phosphorylation [29]. To further test for the impact of MAPK activity on Tipmediated SRE-reporter induction, transfected Jurkat T cells were treated with the MEK inhibitors U0126 and PD0325901. PMA, a chemical diacylglycerol analog known to activate MAPK, was included as a positive control for the inhibitory activity of these reagents (Figure 1). Activation of the SRE reporter by Tip was confirmed, but only partially or non-significantly reduced by U0126 or PD0325901 treatment. In contrast, PMA-induced reporter activity, which was 2.8 -fold higher compared to Tipexpressing cells, was highly sensitive to MEK inhibition (Figure 1A). These data were concordant with ERK1/2 phosphorylation detected by immunoblot analysis. Basal phosphorylation was rather reduced by Tip, enhanced by PMA and suppressed by the inhibitors (Figure 1C). As Tip-induced SRE activity was not accompanied by MAPK activity, we tested an alternative reporter (Figure 1B). The 


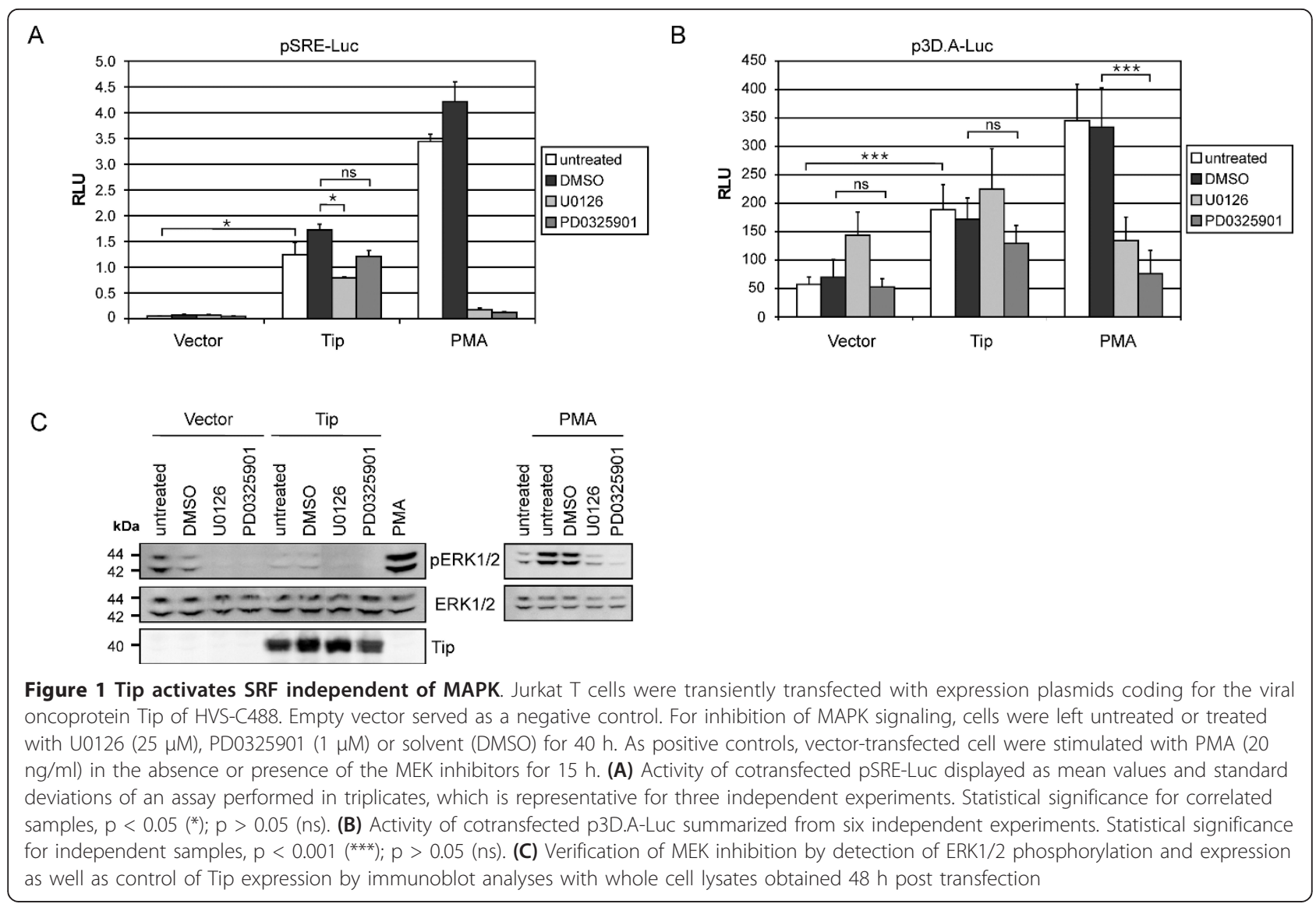

p3D.A luciferase reporter contains a mutated TCF-binding Ets motif within its SRE and is therefore more sensitive to activation by MRTF:SRF complexes. Relative to the SRE reporter, the p3D.A construct displayed a high basal activity in vector-transfected cells. An enhanced activity of this reporter was observed for the MEK inhibitor U0126, but not for PD0325901, indicating off-target functions and restricting the validity of U0126 data. Tip induced a 3-fold increase of the basal activity, and this enhancement was not significantly affected by the MEK inhibitor PD0325901. In contrast, PMA stimulation of vector-transfected cells enhanced the activity about 7 -fold, and this effect was completely abrogated by U0126 and PD0325901. Taken together, the viral oncoprotein Tip induced SRF-responsive luciferase reporters independent of MAPK activity and ERK phosphorylation. Activation of the p3D.A luciferase reporter further points at SRF activation by Tip independent of the MAPK-TCF pathway.

SRF activation involves actin dynamics and the cofactor MAL To corroborate MAPK and, thus, TCF independence of Tip-mediated SRF activation, we next addressed the actinMRTF pathway. To this end, we transfected Jurkat T cells with expression plasmids for wild-type actin (actin wt), an actin polymerization mutant (actinR62D), wild-type full- length MAL (MAL) and a MAL deletion mutant unable to bind actin and SRF (MAL $\triangle \mathrm{N} \triangle \mathrm{B} 1$ ) alone or in combination with Tip (Figure 2A). Expression of the transfected constructs was controlled by immunoblot analysis (Figure $2 B)$. Overexpression of actin, presumably resulting in excess globular actin, diminished the basal and Tipinduced reporter activity by 3.5 - and 2.2-fold, respectively. This effect became more evident when globular actin was enriched by overexpression of actinR62D, which reduced the Tip-induced signal below basal levels. Upon overexpression of MAL, the basal reporter activity was 3.7-fold higher compared to vector alone, and this was further enhanced about 2.5-fold by coexpression of Tip. In contrast, the MAL deletion mutant completely abrogated the signal. To strengthen these observations, we treated transfected cells with Latrunculin B, an inhibitor of actin polymerization and promoter of filamentous actin disassembly. As a positive control we used Cytochalasin D, which binds G-actin irreversibly (Figure 2C). While enrichment of monomeric actin by Latrunculin B inhibited both basal and Tip-induced reporter activity, Cytochalasin D increased the basal activity about 4-fold, but did not further enhance the Tip effect. Thus, actin polymerization and the cofactor MAL indeed play an important role in SRF activation by Tip. 


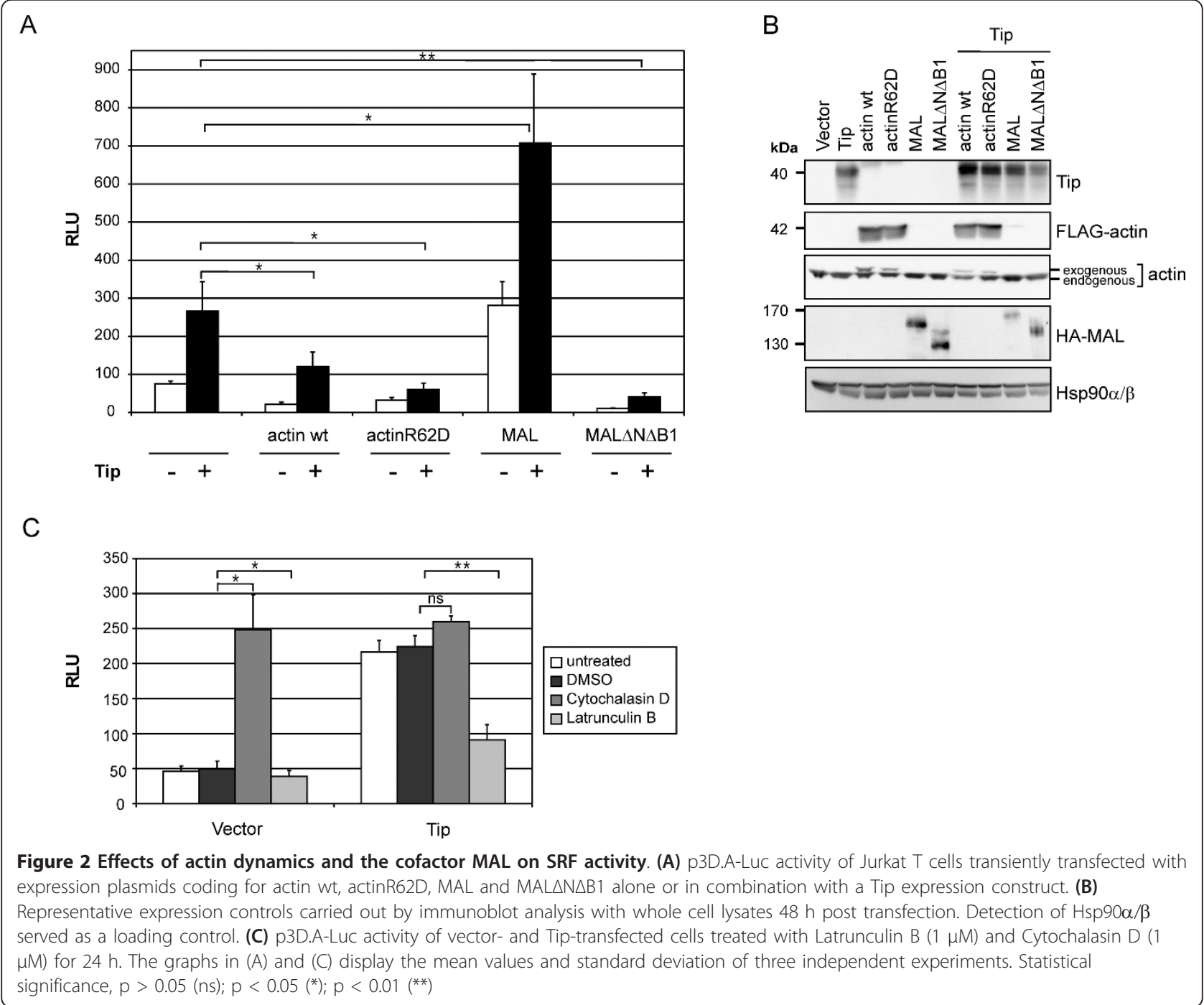

\section{Dominant-negative Rac1 prevents Tip-mediated MAL:SRF activation}

The importance of actin dynamics for Tip-induced SRF activation raised the question whether the small GTPases RhoA, Rac1, Cdc42, inducers of actin polymerization and actin filament stabilization, play a role in this process. Therefore, we used dominant-negative expression constructs for Rac1 (Rac1-T17N) and RhoA (RhoA-T19N) to further elucidate their role in p3D.A reporter induction. Dominant-negative H-Ras (H-RasS17N), a regulator of MAPK and TCFs, was used as a control for interference between the small $G$ proteins (Figure 3A). Expression of the transfected constructs was controlled by immunoblot analysis (Figure $3 \mathrm{~B}$ ). Coexpression of RhoA-T19N and H-Ras-S17N did not significantly reduce Tip-mediated reporter activity. However, overexpression of Rac1-T17N impaired both Tip's effect on the reporter and background activity in vector-transfected cells. Effector pull-down assays to detect GTP-loaded Rac1/2/3 and Cdc42 (GST-PAKCRIB), RhoA (GST-Rhotekin) and H-Ras (GST-RafRBD) suggested an activation of Rac and Cdc42, but not RhoA and H-Ras by Tip (data not shown). However, these findings were not constantly reproducible due to high basal levels of activated Rac1/2/3 and Cdc42 in vector-transfected cells. Nevertheless, the luciferase reporter assays demonstrate a major role of the GTPase Rac1, but not of RhoA and H-Ras, in the actin polymerization- and MAL-dependent SRF activation by Tip.

\section{p3D.A reporter activation by Tip depends on Src-family} kinase interaction and activity

To test for the properties of Tip required to induce SRF activity, we used mutants of Tip defective in its major effector function, the recruitment and activation of the Src-family kinase (SFK) Lck, or carrying substitutions of 
A

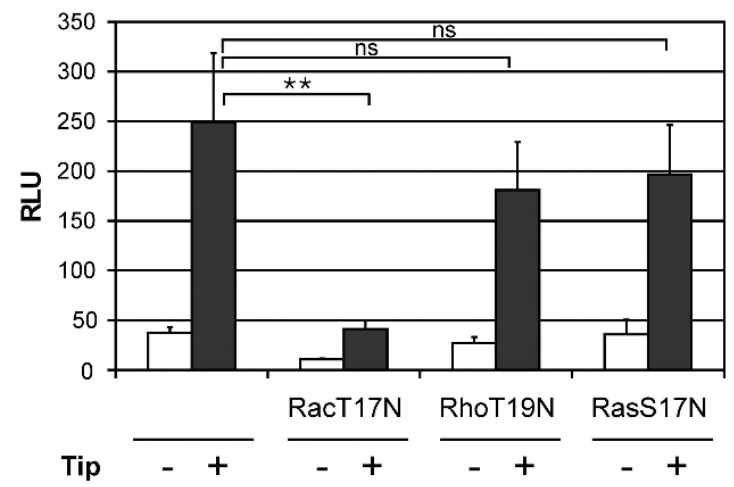

B

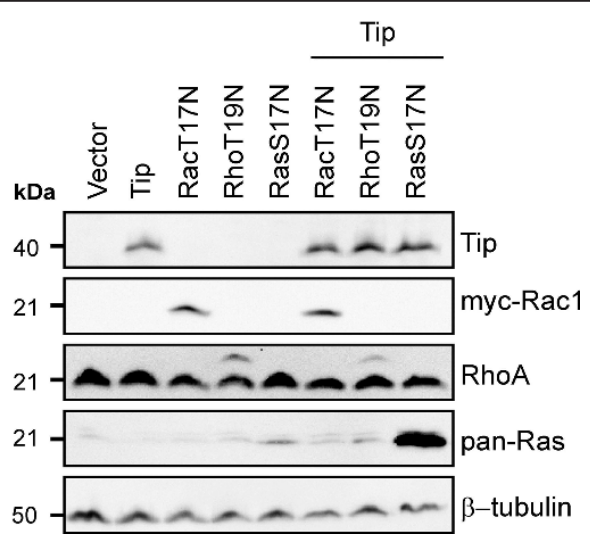

Figure 3 Influence of dominant-negative GTPases on SRF activation. Jurkat T cells were transiently transfected with expression plasmids coding for myc-Rac1-T17N (RacT17N), RhoA-T19N (RhoT19N) and H-Ras-S17N (RasS17N) alone or in combination with a Tip expression construct. (A) Activity of cotransfected p3D.A-Luc displayed as mean values and standard deviation of three independent experiments. Statistical significance, $p>0.05$ (ns); $p<0.01{ }^{* *}$ ). (B) Representative expression control carried out by immunoblot analysis. Detection of $\beta$-tubulin served as a loading control

the conserved tyrosine residues Y114, Y127 and Y155, which may be targets of Lck [22,27,30] (Figure 4A). Expression of the transfected constructs was controlled by immunoblot analysis (Figure 4B). Deletion of the CSKH motif (Tip $\Delta$ CSKH and Tip $\Delta$ CSKHmSH3B) or individual point mutations of tyrosine residues 114 (TipY114F) and 127 (TipY127F) significantly reduced SRF reporter activity to vector levels. The repression observed upon mutation of the SH3 binding motif (TipmSH3B) or tyrosine residue 155 (TipY155F) was not significant. Furthermore, interpretation of the data for TipY127F and TipY155F is restricted by their expression levels, which were reproducibly reduced relative to the wild-type protein. The abolishment of Tipmediated reporter activation by the highly specific SFK inhibitor PP2 verified the requirement of Src-kinase activity (Figure 4C). Immunoblot analysis of protein tyrosine phosphorylation monitored a modulating function of Tip and the inhibitory efficacy of PP2 (Figure 4D). Hence, Tip relies on both, Lck interaction and SFK activity, to trigger MAL:SRF reporter activity. Furthermore, tyrosine residues Y114 and Y127, known to be critical for STAT3 activation [28] and IL-2-independent $\mathrm{T}$-cell transformation [22], respectively, likely contribute to Tip-induced SRF activity.

\section{TCR stimulation induces p3D.A reporter activity}

The viral oncoprotein Tip activated SRF in T cells via the actin-regulated cofactor MAL (Figure 2), while previous reports demonstrated SRF activation via the MEK-ERK pathway in response to TCR stimulation of Jurkat T cells and in mouse T-cell development [13,31]. This discrepancy prompted us to assess whether TCR stimulation alone can trigger the p3D.A luciferase reporter or further enhance the Tip effect (Figure 5A). TCR and coreceptor engagement via CD3/CD28 antibodies resulted in a 10 -fold enhanced reporter activity in vector-transfected Jurkat $\mathrm{T}$ cells relative to unstimulated cells. In contrast, CD3/CD28 antibody treatment did not significantly augment the Tiptriggered signal. As ERK phosphorylation was absent in Tip-transfected cells (Figure 5B), this lack of cooperation correlated with an impaired CD3/CD28-induced signaling, which is in accordance with suppression of TCR signaling by Tip [32]. In order to specify the TCR-triggered pathway involved, CD3/CD28-stimulated and unstimulated vectortransfected cells were treated with inhibitors of SFK (PP2), MEK (PD0325901), and actin polymerization (Latrunculin B) (Figure 5C). TCR-induced reporter activity was significantly reduced in all treated samples. All three inhibitors were similarly effective, with low but significant residual activities relative to unstimulated cells. Unexpectedly, the residual activities in PD0325901- and Latrunculin B-treated cells did not add up to the activity of solvent-treated cells (DMSO). This finding may be related to the partial reduction of ERK phosphorylation by Latrunculin B (Figure 5D). The impact of actin polymerization on SRF activation in $\mathrm{T}$ cells was further addressed by the expression of constitutively active Rac1 (Rac1-G12V) and RhoA (RhoA-Q63L) in the Jurkat system (Figure 5E). Rac1-G12V and RhoA-Q63L (Figure 5E) were equally effective and even more potent than $\mathrm{CD} 3 / \mathrm{CD} 28$ stimulation (Figure $5 \mathrm{~A}, \mathrm{C}$ ) in inducing 3D.A reporter activity. In conclusion, TCR stimulation relied on both, MAPK signaling and actin polymerization, to activate SRF.

\section{Discussion}

Our study revealed that the oncoprotein Tip of Herpesvirus saimiri (HVS) activates the serum response factor 


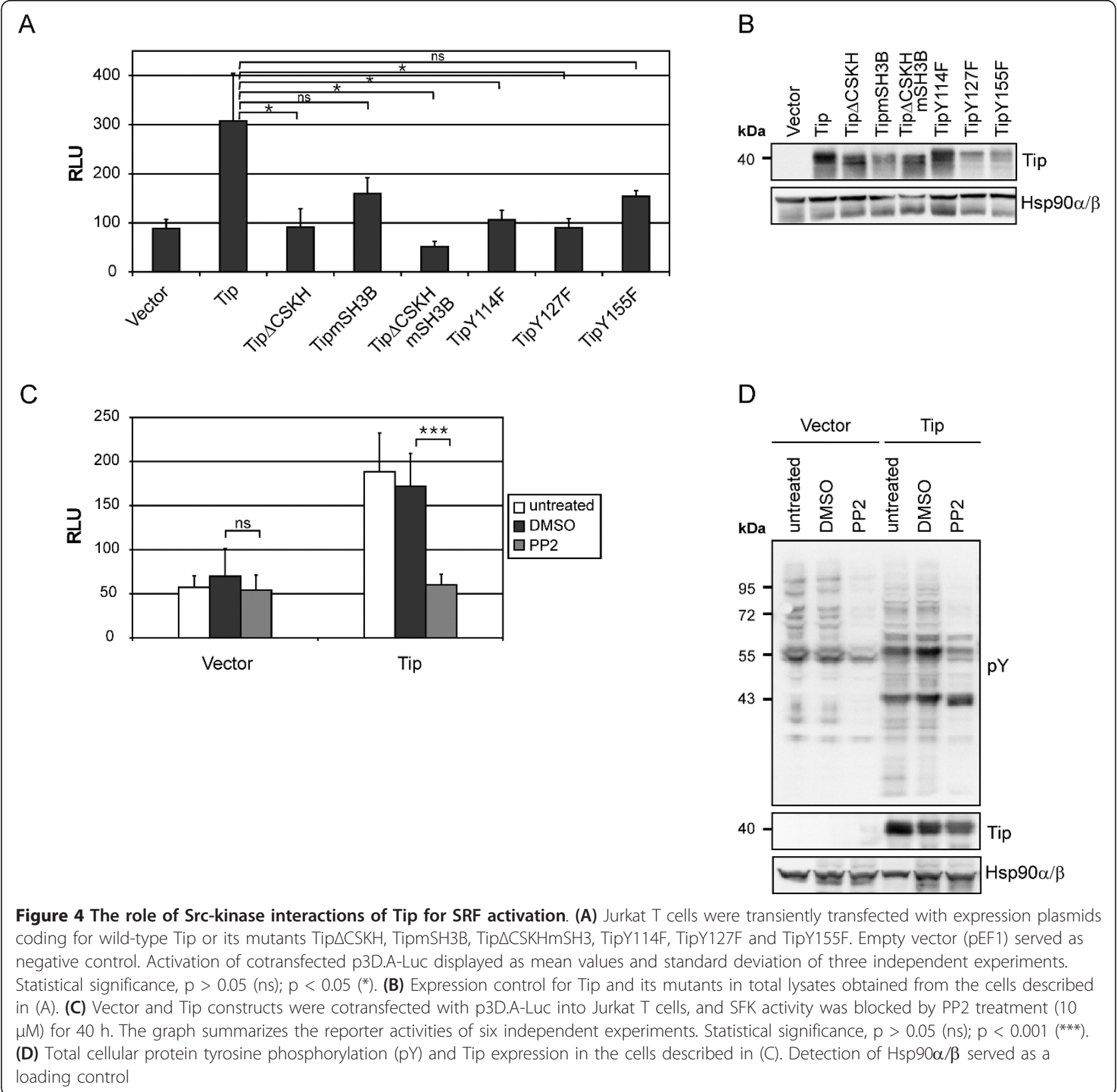

(SRF) in T cells. This activation mainly depends on actin-mediated MRTF coactivation, with minor contributions of MEK-mediated TCF coactivation. Discrimination of coactivator involvement was assessed using two SRF-dependent luciferase reporter constructs, based on the $c$-fos SRE, considered to be specific for TCF coactivation, and on a mutated SRE (3D.A), considered to respond preferentially to MRTF coactivation. However, largely MEK-independent SRE activation by Tip and MEK-sensitive 3D.A activation by PMA revealed a restricted specificity of the reporters in the Jurkat $\mathrm{T}$ cells used throughout this study. Hence, we included chemical inhibitors and overexpression of mutant signaling intermediates to assign Tip-induced SRF activation to the actin-dependent MRTF coactivation pathway. Targeting of this pathway by a viral $\mathrm{T}$-cell oncoprotein was unexpected, as SRF function in T cells had previously been linked mainly to the TCF pathway [13].

SRF activation in our system strictly relied on the ability of Tip to engage Lck. This interaction is reported to result in kinase activation [25,33-35], which is also wellknown as an initial step in T-cell activation. Indeed, independent of Tip, CD3/CD28 stimulation triggered the 3D. A reporter through Src-family kinase (SFK) activity and both, TCF and MRTF pathways. These findings are in 


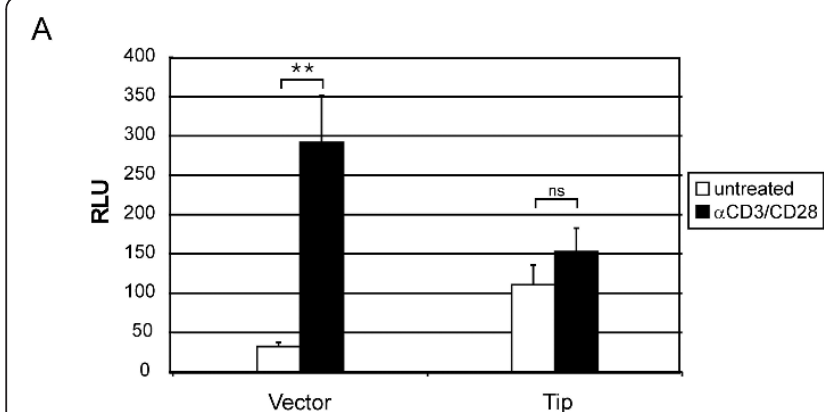

C

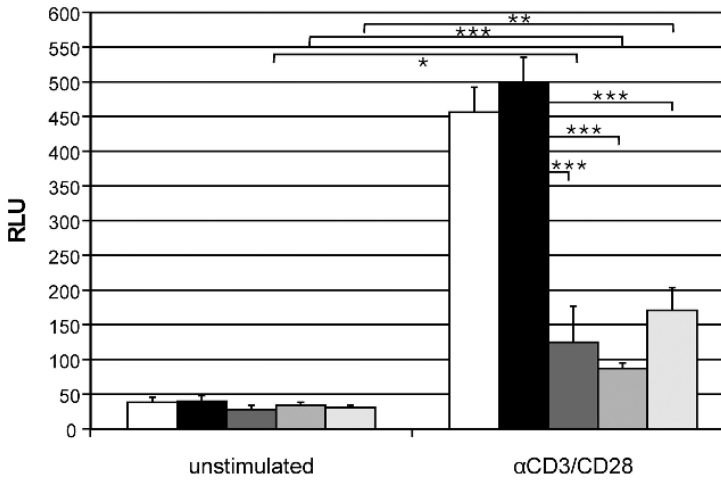

E

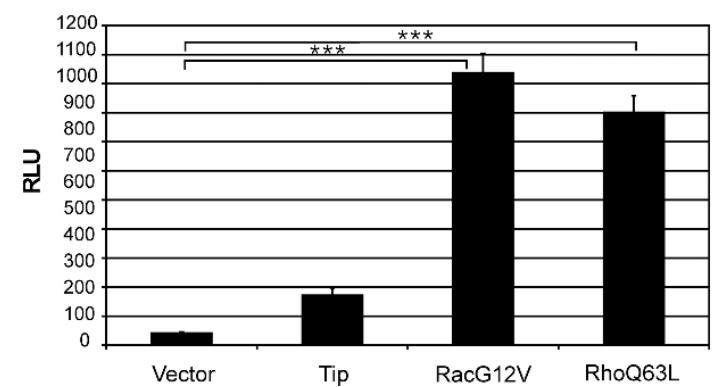

B

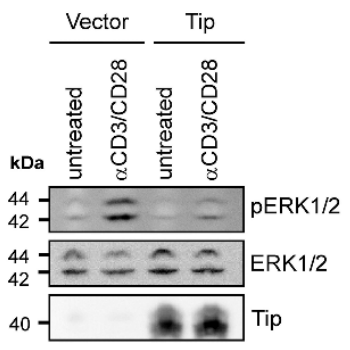

D

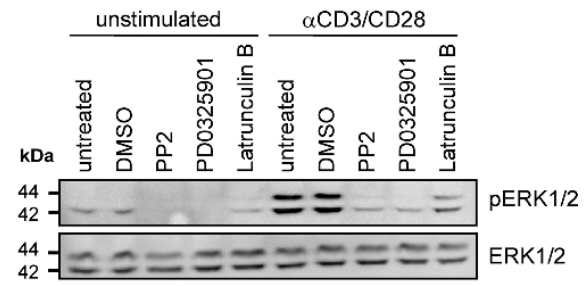

Figure 5 SRF activation by TCR engagement and by constitutively active Rac1 or RhoA. (A) Jurkat T cells were transiently transfected with p3D.A-Luc and vector or expression plasmids coding for Tip and stimulated for $14 \mathrm{~h}$ in 6 -well plates coated with antibodies directed against CD3 and CD28 ( $\alpha$ CD3/CD28). Reporter activation is summarized as mean values and standard error of five independent experiments. Statistical significance, $p>0.05$ (ns); $\left.p<0.01{ }^{(*}\right)$. (B) Representative immunoblots for the cells used in (A) displaying ERK1/2 phosphorylation and expression as well as Tip expression. (C) Jurkat T cells were transiently transfected with p3D.A-Luc and vector. Cells were treated with PP2 (10

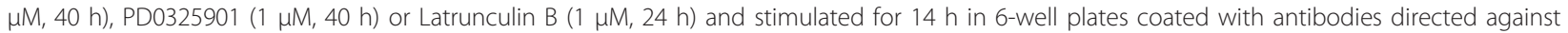
CD3 and CD28 ( $\alpha C D 3 / C D 28)$. Reporter activation is summarized as mean values and standard deviation of three independent experiments. Statistical significance, $\mathrm{p}<0.05\left(^{*}\right) ; \mathrm{p}<0.01\left(^{* *}\right) ; \mathrm{p}<0.001\left(^{(* *}\right)$. (D) Representative immunoblots for the cells described in (C) displaying ERK1/2 phosphorylation and expression. (E) Vector and expression plasmids coding for Tip, myc-Rac1-G12V (RacG12V) or myc-RhoA-Q63L (RhoQ63L) were cotransfected with p3D.A-Luc. The graph summarizes data of five independent experiments. Statistical significance, $p<0.001$ (***). (F) Representative immunoblots displaying myc-Rac1-G12V, myc-RhoA-Q63L and Tip expression. Detection of $\beta$-tubulin served as a loading control

accordance with early results on SRE-dependent transcription in Jurkat T cells [31]. Thus, Lck-dependent MRTF coactivation, which we suggest for Tip, may as well apply to T-cell stimulation. However, while Tip triggers SRF largely independent of MAPK activity, stimulation-induced SRF activation substantially involves MAPK signaling and likely integrates different intracellular signaling routes. The interference of Tip with receptor- mediated SRF activation most likey occurs further upstream. Dependent on its localization in lipid rafts, Tip induces the internalization of TCR complexes [36-38]. Independent of its lipid raft association, Tip blocks TCRmediated intracellular signaling most likely through sequestration of Lck $[32,38]$. Consequently, Tip-expressing cells are refractory to receptor ligation by stimulating antibodies. 
The dependence of Tip-induced SRF activation on Lck interaction, Src-family kinase (SFK) activity and the potential Lck phosphorylation sites in Tip, Y114 and Y127, draws the attention to the Tip:Lck effectors involved in this pathway. So far, only STATs, especially STAT3, are described as direct targets of Tip-activated Lck [21,24-27]. Tip-induced STAT3 activation depends on residue Y114, which is not required for human Tcell transformation in vitro [28]. However, the potential of STAT3 to promote invasion in various cancers [23] may well relate to the massive tissue invasion by HVSlymphoma cells [26,39], which is not reflected in the cell culture system. Therefore, while effectors of Tip essential for viral T-cell transformation are still not identified, we suggest that Tip Y114 contributes to viral oncogenesis through STAT3-regulated lymphocyte invasion. In this context, STAT3 would be expected as an upstream regulator of RhoGTPases. However, an emerging model positions STAT3 downstream of Rac1 and Cdc42 in the regulation of cell proliferation and migration [40]. Alternatively, transcriptional regulation of genes involved in MRTF:SRF activation by Tip-induced STAT3 appears conceivable. Such an indirect mechanism might also be elicited by STAT5, a recently identified target of Tip [41] likely related to the strict IL-2 dependence of viral transformation in the presence of TipY127F [22]. In any case, a functional link between STAT3 or STAT5 and MRTF:SRF, to the best of our knowledge, has not been reported. Hence, Tip-activated Lck may trigger SRF activation through alternative, yet unknown effectors like the various RhoGTPase guanine nucleotide exchange factors (GEFs) expressed in T cells [42]. Altogether, mechanisms of MRTF:SRF activation proximal to the Tip:Lck complex remain to be established.

The RhoGTPases RhoA, Rac1 and Cdc42 directly regulate actin cytoskeleton organization [7] and therefore share the potential to modulate cellular G-actin pools, which in turn determine MRTF coactivator availability [1]. We expressed constitutively active Rac1 and RhoA and thereby proved the inducibility of MRTF:SRF by both GTPases in T cells independent of Tip. Dominantnegative versions of Rac1, RhoA and Ras were used to test for the involvement of these GTPases in Tipmediated SRF activation. The missing influence of dominant-negative Ras corroborated the TCF independence of Tip-induced SRF activation. Suppression of the Tip effect by inhibitory Rac1 and not RhoA is in contrast to the initial report on SRF activation by MAL in NIH3T3 fibroblasts [8], but in accordance with MAL signaling in epithelial cells [43]. We assumed that Tip induces SRF via Rac1, but not RhoA. Accordingly, active (GTPbound) RhoA and H-Ras were not detected in Tipexpressing cells, whereas cellular levels of basally active Rac1 and Cdc42 were enhanced by Tip in some, but not all effector pull-down assays performed. We further used the Rac1/Cdc42-glucosylating $C$. difficile toxins that have been shown to inhibit SRF activation induced by $\mathrm{Ca}^{2+}$-dependent dissociation of epithelial integrity [43]. Unexpectedly, the C. difficile toxins failed to suppress Tip-induced reporter activity in our Jurkat system (data not shown). This observation is apparently inconsistant with our observation that Rac1-T17N strongly reduces Tip-induced SRF activation. In general, either pronounced Rac1/Cdc42 activation or pronounced Rac1/Cdc42 phosphorylation by Akt1 protects Rac1/ Cdc42 from toxin-catalyzed glucosylation and inactivation $[44,45]$. In particular, protective phosphorylation of Rac1/Cdc42 has to be taken into account, as Jurkat T cells are deficient in expression of PTEN, a major negative regulator of PI3K/Akt signaling [46]. Based on the data available, we would exclude RhoA and Ras and suggest Rac1 and Cdc42 activation in response to Tip expression as the crucial step in SRF induction. The mechanism of the Tip-mediated activation of Rac1/ Cdc42, however, remains to be clarified.

Besides the critical role of Rac1 in Tip-induced SRF activation, our results substantiate an essential role of actin and actin-regulated MRTF in SRF activation by Tip in T cells. The syngergism between ectopic MAL and the viral oncoprotein, which is in contrast to the effects obtained with the cellular oncoprotein OTTMAL [47], points at limiting MAL expression levels and clearly positions Tip upstream in the activation cascade. However, although we used wild-type and mutant MAL expression constructs, our assays are not suited to discriminate the contribution of the individual MRTFfamily proteins, MAL/MRTF-A and MRTF-B, which may add another layer of complexity to SRF regulation.

MRTF:SRF functions in $T$ cells are not characterized yet, and $\mathrm{T}$ cell-specific target genes of this transcription factor complex are not known. However, transcription of cytoskeletal regulators like MYH9 and MYL9 is elevated in different non-lymphoid cancer cell lines, which depend on MRTFs and SRF for cell spreading, adhesion, and motility [48]. Thus, MRTF:SRF activation by Tip, a viral oncoprotein essential for the development of fulminant $\mathrm{T}$-cell lymphoma characterized by infiltration of multiple organs $[16,26]$, may well contribute to viral oncogenesis and tissue invasion of tumor cells.

\section{Conclusion}

Our study on cellular signaling by the viral oncoprotein Tip demonstrates SRF coactivation by MRTFs and not TCFs in T cells. MRTF:SRF induction depended on actin polymerization and RhoGTPase activity as well as Tip:Lck interaction and SFK activity (Figure 6). Furthermore, our data hint at MRTF:SRF activation by TCR stimulation independent of Tip. Future studies will have to 


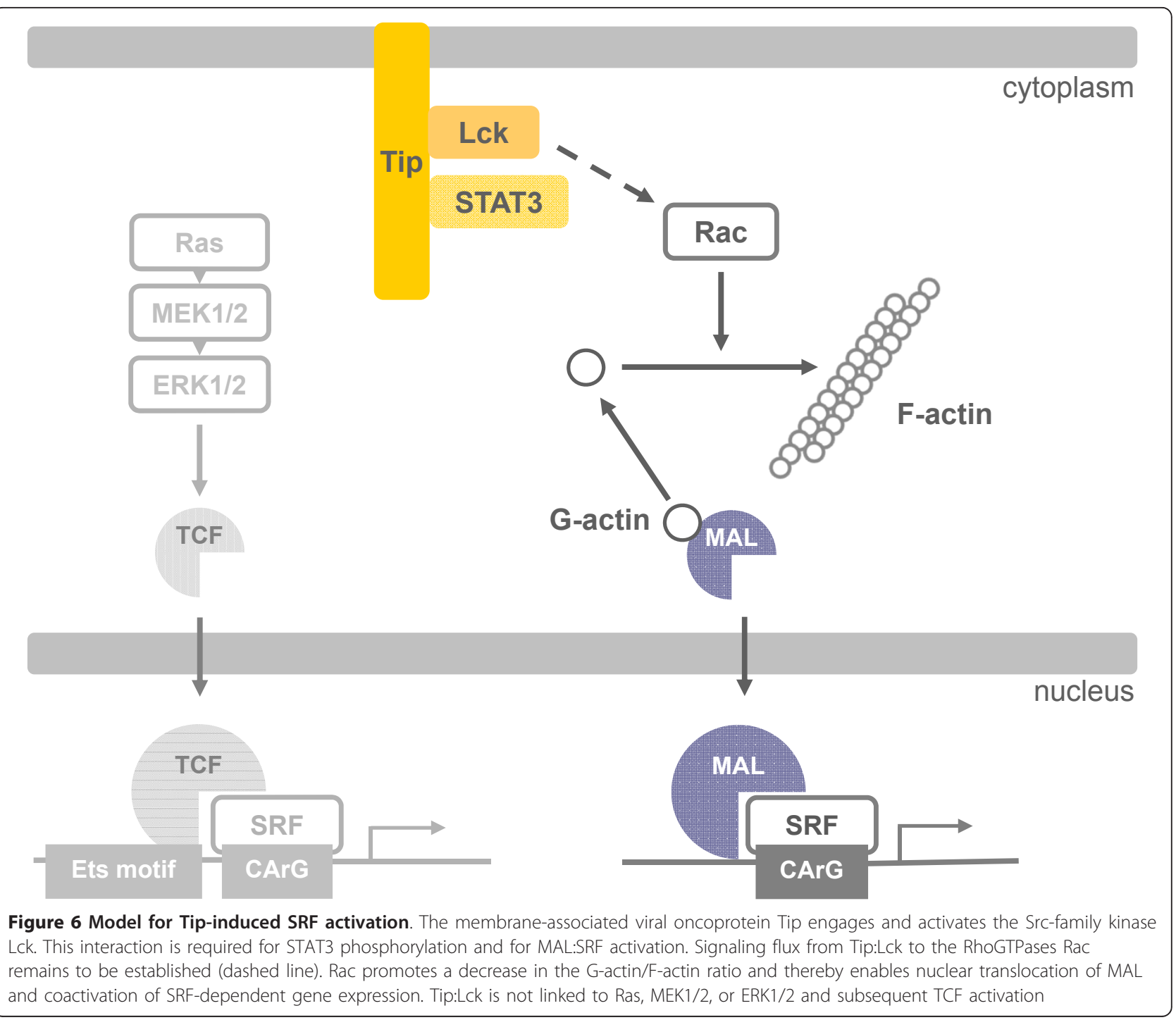

reveal the detailed mechanisms and target genes of the pathway triggered by Tip as well as its applicability to $\mathrm{T}$ cells in general. This approach is anticipated to resolve the functional relevance of MRTF:SRF activity in T-cell regulation and in viral oncogenesis.

\section{Methods}

\section{Cell culture}

Jurkat T cells (E6.1, ATCC TIB-152) were cultured in RPMI 1640 medium supplemented with $10 \%$ fetal calf serum (FCS), glutamine $(350 \mu \mathrm{g} / \mathrm{ml})$ and gentamicin $(100 \mu \mathrm{g} / \mathrm{ml})$ at a maximum concentration of $0.5-1 \times 10^{6}$ cells $/ \mathrm{ml}$.

\section{Transient transfection of Jurkat T cells}

Transfection of $5-10 \times 10^{6}$ cells $/ \mathrm{ml}$ Jurkat $\mathrm{T}$ cells was carried out by electroporation in medium without antibiotics at $250 \mathrm{~V}, 1,500 \mu \mathrm{F}$ using a Gene pulser $\times$ cell $^{\mathrm{TM}}$
Electroporation System (Bio-Rad). For each sample, a total of $50 \mu \mathrm{g}$ plasmid DNA was used and appropriate empty vector was included to equalize plasmid DNA amounts. Transfected cells, cultured in complete medium without antibiotics, were harvested after $48 \mathrm{~h}$, washed with phosphate-buffered saline (PBS) and processed for luciferase reporter gene assays or immunoblot analysis.

\section{Expression plasmids}

Jurkat T cells were transfected with $20 \mu \mathrm{g}$ of expression constructs coding for wild type and mutants of the viral oncoprotein Tip derived from HVS-C488: pEF1-Tip, pEF1-Tip $\Delta$ CSKH, pEF1-TipmSH3B, pEF1-Tip $\Delta$ CSKHm SH3B, pEF1-TipY114F, pEF1-TipY127F, pEF1-TipY155F [49]. All Tip constructs are N-terminally myc-tagged. The expression plasmids pEF-FLAG-actin wt; pEF-FLAGactinR62D, coding for a FLAG-tagged polymerization 
mutant of actin; pEF-MAL-HA (f.l.), encoding HA-tagged full-length murine MAL; pEF-MAL $\triangle \mathrm{N} \triangle \mathrm{B} 1-\mathrm{HA}$, coding for a MAL deletion mutant unable to bind to actin and SRF, were described previously $[8,50]$. Sequences coding for dominant-negative Rac1 (RacT17N) and RhoA (RhoT19N) and constitutively active Rac1 (RacG12V) and RhoA (RhoQ63L) were amplified by PCR with oligonucleotide primers introducing terminal BamHI and EcoRI restriction sites and a N-terminal myc-tag (myc-RacT17N, myc-RacG12V, myc-RhoQ63L) (primers available upon request) were cloned into pEF1 to yield the expression constructs pEF1-myc-RacT17N, pEF1-RhoT19N, pEF1myc-RacG12V and pEF1-myc-RhoQ63L. Dominant-negative Ras was expressed using the plasmid pcDNA3RasS17N (kindly provided by A. Wittinghofer, Dortmund, Germany). Integrity of the coding sequences was confirmed by automated DNA sequencing (ABI 3130, Applied Biosystems).

\section{Immunoblot analysis}

Jurkat $\mathrm{T}$ cells were lysed in RIPA buffer and processed as previously described to generate whole cell lysates [29]. Protein extracts of $0.5-1 \times 10^{6}$ Jurkat T cells were loaded on SDS-polyacrylamide gels and transferred to polyvinylidene difluoride membranes (GE Healthcare). After blocking with $5 \%$ milk powder in $0.1 \%$ Tween 20 PBS or NET-gelatine $(150 \mathrm{mM} \mathrm{NaCl} ; 5 \mathrm{mM}$ EDTA; 50 $\mathrm{mM}$ Tris- $\mathrm{HCl} \mathrm{pH} 7.5 ; 0.05 \%$ TritonX-100; $2.5 \mathrm{~g} / \mathrm{ml}$ gelatine), the membranes were probed with antibodies directed against: phosphotyrosine (4 G10, Millipore), pERK1/ 2 (pY204 in ERK1), Hsp90 $\alpha / \beta$ (Santa Cruz), ERK1/2, RhoA, Rac1/2/3 (Cell Signaling Technology), Pan-Ras (Calbiochem), Tip [17], Myc-epitope (9E10; ATCC CRL1729), FLAG-epitope (M2, HRP-coupled; Sigma), HAepitope (Convance), $\beta$-tubulin (GE Healthcare). Binding of primary antibodies was detected using horseradish peroxidase-coupled secondary antibodies directed against mouse or rabbit immunoglobulins (Dako). Primary and secondary antibodies were diluted in blocking buffer. Immunodetection was performed by chemiluminescence and documented with a Kodak Image Station 4000 MM PRO camera.

\section{Luciferase reporter gene assay}

Jurkat T cells were transfected with $20 \mu \mathrm{g}$ of the individual effector plasmids and $10 \mu \mathrm{g}$ of the reporter plasmid pSRE-luc containing five SRE of the c-fos promoter (Stratagene) or p3D.A-Luc [51] comprising three SRE with a mutated Ets motif. Cells were harvested $48 \mathrm{~h}$ post transfection and divided equally for luciferase activity quantification and immunoblots. For luciferase reporter gene assay, cells were lysed and luminescence intensity was measured as described [52]. Raw data were normalized to the protein content of each sample as determined by a BCA assay (Uptima) and indicated as relative light units (RLU). Data were statistically evaluated with two-tailed t-tests for correlated (Figure 1A) or independent (all other figures) samples using the onlinetools provided by the VassarStats Website for Statistical Computation [http://faculty.vassar.edu/lowry/VassarStats.html]. Results were assigned to the categories $\mathrm{p}>$ 0.05 (ns, not significant), $\mathrm{p}<0.05\left(^{(*)}, \mathrm{p}<0.01\left(^{(* *)}\right), \mathrm{p}<\right.$ $0.001{ }^{(* * *)}$.

\section{Inhibitor treatment and $\mathrm{CD} 3 / \mathrm{CD} 28$ ligation}

For inhibitor treatment, transfected Jurkat $\mathrm{T}$ cells were seeded in a 12-well plate at a density of approximately $0.5 \times 10^{6}$ cells $/ \mathrm{ml}$. The SFK inhibitor PP2 (Sigma; 10 $\mu \mathrm{M})$ and the MAPK inhibitors U0126 (Biomol Germany; $25 \mu \mathrm{M})$ and PD0325901 (1 $\mu \mathrm{M})$ were added $8 \mathrm{~h}$ post transfection and remained in the cultures until harvesting of the cells. 12-O-tetradecanoylphorbol-13-acetate (PMA; Sigma; $20 \mathrm{ng} / \mathrm{ml}$ ), combined with MAPK inhibitors if applicable, was added for $15 \mathrm{~h}$. To modulate actin polymerization, cells were treated with Latrunculin B (Calbiochem; $1 \mu \mathrm{M}$ ), Cytochalasin D (Applichem; 1 $\mu \mathrm{M})$ for $24 \mathrm{~h}$. Under these conditions all inhibitors were not toxic to Jurkat $\mathrm{T}$ cells as measured by propidiumiodide staining and flow cytometry. T cell-receptor stimulation of transfected Jurkat $\mathrm{T}$ cells was carried out for $14 \mathrm{~h}$ in a 6 -well plate at a density of approximately $1 \times$ $10^{6}$ cells $/ \mathrm{ml}$ previously coated with antibodies against CD3 (OKT3; Janssen-Cilag; $10 \mu \mathrm{g} / \mathrm{ml}$ ) and CD28 (a gift from R. Kroczek, Robert Koch-Institut, Berlin; $5 \mu \mathrm{g} / \mathrm{ml}$ ).

\begin{abstract}
Abbreviations
CSKH: Sequence homologous to the C-terminus of Src-family kinase domains; ERK: Extracellular signal-regulated kinase; G-actin: Globular actin; GEF: Guanine nucleotide exchange factor; GTP: Guanosine triphosphate; HVS: Herpesvirus saimiri; MAPK: Mitogen-activated protein kinase; MRTFs:

Myocardin-related transcription factors; MYH9: Myosin heavy chain 9: non muscle; MYL9: Myosin light chain 9: regulatory; PMA: 12-O-

tetradecanoylphorbol-13-acetate; RhoGTPases: Guanosine triphosphatases of the Rho family; SFK: Src-family kinase; SH3: Src homology domain 3 binding sequence; SRE: Serum response element; SRF: Serum response factor; STAT: Signal transducer and activator of transcription; StpC: Saimiri transformationassociated protein of subgroup C; TCF: Ternary complex factor; Tip: Tyrosine interacting protein.
\end{abstract}

\section{Acknowledgements}

We thank Richard Kroczek (Berlin) and Alfred Wittinghofer (Dortmund) for kindly providing antibodies and plasmids. This work was supported by grants GRK1071/C3 and Bi465/5-1 from the German Research Foundation (DFG) and by the BIGSS program (State of Bavaria).

\section{Author details}

${ }^{1}$ Institut für Klinische und Molekulare Virologie, Friedrich-AlexanderUniversität Erlangen-Nürnberg, Erlangen, Germany. ${ }^{2}$ Institut für Toxikologie, Medizinische Hochschule Hannover, Hannover, Germany. ${ }^{3}$ Department Molecular Biology, Max-Planck-Institut für Biochemie, Munich, Germany.

\section{Authors' contributions}

All authors have read and approved the final manuscript. BB conceived and approved the experiments. KK and JS performed the experiments. KK, SJdJ, 
JCA, JS, HG, GP and BB analyzed and interpreted the data. BB and KK drafted the manuscript.

\section{Competing interests}

The authors declare that they have no competing interests.

Received: 20 January 2012 Accepted: 3 March 2012

Published: 3 March 2012

\section{References}

1. Posern G, Treisman R: Actin' together: serum response factor, its cofactors and the link to signal transduction. Trends Cell Biol 2006, 16:588-596.

2. Miano JM: Role of serum response factor in the pathogenesis of disease. Lab Invest 2010, 90:1274-1284.

3. Shaw PE, Schroter $H$, Nordheim A: The ability of a ternary complex to form over the serum response element correlates with serum inducibility of the human c-fos promoter. Cell 1989, 56:563-572.

4. Treisman R: Ternary complex factors: growth factor regulated transcriptional activators. Curr Opin Genet Dev 1994, 4:96-101.

5. Treisman R: Journey to the surface of the cell: fos regulation and the SRE. EMBO J 1995, 14:4905-4913.

6. Olson EN, Nordheim A: Linking actin dynamics and gene transcription to drive cellular motile functions. Nat Rev Mol Cell Biol 2010, 11:353-365

7. Spiering D, Hodgson L: Dynamics of the Rho-family small GTPases in actin regulation and motility. Cell Adh Migr 2011, 5:170-180.

8. Miralles F, Posern G, Zaromytidou Al, Treisman R: Actin dynamics control SRF activity by regulation of its coactivator MAL. Cell 2003, 113:329-342.

9. Wang Z, Wang DZ, Hockemeyer D, McAnally J, Nordheim A, Olson EN: Myocardin and ternary complex factors compete for SRF to control smooth muscle gene expression. Nature 2004, 428:185-189.

10. Zaromytidou Al, Miralles F, Treisman R: MAL and ternary complex factor use different mechanisms to contact a common surface on the serum response factor DNA-binding domain. Mol Cell Biol 2006, 26:4134-4148.

11. Gineitis $D$, Treisman R: Differential usage of signal transduction pathways defines two types of serum response factor target gene. J Biol Chem 2001, 276:24531-24539.

12. Fleige A, Alberti $S$, Grobe L, Frischmann U, Geffers R, Muller W, Nordheim A, Schippers A: Serum response factor contributes selectively to lymphocyte development. J Biol Chem 2007, 282:24320-24328.

13. Mylona A, Nicolas R, Maurice D, Sargent M, Tuil D, Daegelen D, Treisman R, Costello P: The essential function for serum response factor in T-cell development reflects its specific coupling to extracellular signalregulated kinase signaling. Mol Cell Biol 2011, 31:267-276.

14. Ensser A, Fleckenstein B: T-cell transformation and oncogenesis by gamma2-herpesviruses. Adv Cancer Res 2005, 93:91-128.

15. Biesinger B, Müller-Fleckenstein I, Simmer B, Lang G, Wittmann S, Platzer E, Desrosiers RC, Fleckenstein B: Stable growth transformation of human T lymphocytes by herpesvirus saimiri. Proc Natl Acad Sci USA 1992, 89:3116-3119.

16. Duboise SM, Guo J, Czajak S, Desrosiers RC, Jung JU: STP and Tip are essential for herpesvirus saimiri oncogenicity. J Virol 1998, 72:1308-1313.

17. Wehner LE, Schröder N, Kamino K, Friedrich U, Biesinger B, Rüther U: Herpesvirus saimiri Tip gene causes T-cell lymphomas in transgenic mice. DNA Cell Biol 2001, 20:81-88.

18. Biesinger $B$, Tsygankov AY, Fickenscher $H$, Emmrich F, Fleckenstein $B$, Bolen JB, Bröker BM: The product of the Herpesvirus saimiri open reading frame 1 (tip) interacts with T cell-specific kinase $\mathrm{p} 56^{\mathrm{lck}}$ in transformed cells. J Biol Chem 1995, 270:4729-4734.

19. Smith-Garvin JE, Koretzky GA, Jordan MS: T cell activation. Annu Rev Immunol 2009, 27:591-619.

20. Jung JU, Lang SM, Friedrich U, Jun T, Roberts TM, Desrosiers RC, Biesinger B: Identification of Lck-binding elements in tip of herpesvirus saimiri. J Biol Chem 1995, 270:20660-20667.

21. Lund TC, Prator PC, Medveczky MM, Medveczky PG: The Lck binding domain of herpesvirus saimiri tip-484 constitutively activates Lck and STAT3 in T cells. J Virol 1999, 73:1689-1694.

22. Heck E, Friedrich U, Gack MU, Lengenfelder D, Schmidt M, MüllerFleckenstein I, Fleckenstein B, Ensser A, Biesinger B: Growth transformation of human $T$ cells by herpesvirus saimiri requires multiple Tip-Lck interaction motifs. J Virol 2006, 80:9934-9942.
23. Yu H, Pardoll D, Jove R: STATs in cancer inflammation and immunity: a leading role for STAT3. Nat Rev Cancer 2009, 9:798-809.

24. Lund TC, Garcia R, Medveczky MM, Jove R, Medveczky PG: Activation of STAT transcription factors by herpesvirus Saimiri Tip-484 requires p56lck. J Virol 1997, 71:6677-6682.

25. Kjellen P, Amdjadi K, Lund TC, Medveczky PG, Sefton BM: The herpesvirus saimiri tip484 and tip488 proteins both stimulate Ick tyrosine protein kinase activity in vivo and in vitro. Virology 2002, 297:281-288.

26. Reiss $C$, Niedobitek G, Hör S, Lisner R, Friedrich U, Bodemer W, Biesinger B: Peripheral T-cell lymphoma in herpesvirus saimiri-infected tamarins: tumor cell lines reveal subgroup-specific differences. Virology 2002, 294:31-46

27. Hartley DA, Cooper GM: Direct binding and activation of STAT transcription factors by the herpesvirus saimiri protein tip. J Biol Chem 2000, 275:16925-16932

28. Heck E, Lengenfelder D, Schmidt M, Müller-Fleckenstein I, Fleckenstein B, Biesinger B, Ensser A: T-cell growth transformation by herpesvirus saimiri is independent of STAT3 activation. J Virol 2005, 79:5713-5720.

29. Glanz A, Albrecht JC, Heinemann S, Fleckenstein B, Isakov N, Biesinger B: NF-kappaB activation by the viral oncoprotein StpC enhances IFNgamma production in T cells. Immunol Cell Biol 2008, 86:622-630.

30. Ensser A, Thurau M, Wittmann S, Fickenscher $\mathrm{H}$ : The genome of herpesvirus saimiri C488 which is capable of transforming human T cells. Virology 2003, 314:471-487.

31. Charvet C, Auberger P, Tartare-Deckert S, Bernard A, Deckert M: Vav1 couples $T$ cell receptor to serum response factor-dependent transcription via a MEK-dependent pathway. J Biol Chem 2002, 277:15376-15384.

32. Cho NH, Feng $P$, Lee $S H$, Lee BS, Liang $X$, Chang H, Jung JU: Inhibition of T cell receptor signal transduction by tyrosine kinase-interacting protein of Herpesvirus saimiri. J Exp Med 2004, 200:681-687.

33. Wiese N, Tsygankov AY, Klauenberg U, Bolen JB, Fleischer B, Bröker BM: Selective activation of $\mathrm{T}$ cell kinase p56lck by herpesvirus saimiri protein tip. J Biol Chem 1996, 271:847-852.

34. Lund T, Medveczky MM, Medveczky PG: Herpesvirus saimiri Tip-484 membrane protein markedly increases p56lck activity in T cells. J Virol 1997, 71:378-382.

35. Hartley DA, Hurley TR, Hardwick JS, Lund TC, Medveczky PG, Sefton BM: Activation of the Lck Tyrosine-protein Kinase by the binding of the tip protein of herpesvirus Saimiri in the absence of regulatory tyrosine phosphorylation. J Biol Chem 1999, 274:20056-20059.

36. Park J, Lee BS, Choi JK, Means RE, Choe J, Jung JU: Herpesviral protein targets a cellular WD repeat endosomal protein to downregulate $T$ lymphocyte receptor expression. Immunity 2002, 17:221-233.

37. Park J, Cho NH, Choi JK, Feng P, Choe J, Jung JU: Distinct roles of cellula Lck and p80 proteins in herpesvirus saimiri Tip function on lipid rafts. $J$ Virol 2003, 77:9041-9051.

38. Cho NH, Kingston D, Chang H, Kwon EK, Kim JM, Lee JH, Chu H, Choi MS, Kim IS, Jung JU: Association of herpesvirus saimiri tip with lipid raft is essential for downregulation of T-cell receptor and CD4 coreceptor. $J$ Virol 2006, 80:108-118.

39. Fleckenstein B, Desrosiers RC: Herpesvirus saimiri and Herpesvirus ateles. In The Herpesviruses. Edited by: Roizman B. New York: Plenum Publishing Corporation; 1982:253-332.

40. Raptis L, Arulanandam R, Geletu M, Turkson J: The R(h)oads to Stat3: Stat3 activation by the Rho GTPases. Exp Cell Res 2011, 317:1787-1795.

41. Katsch K, de Jong SJ, Schmidt M, Müller-Fleckenstein I, Fleckenstein B, Albrecht JC, Biesinger B: Species restriction of Herpesvirus saimiri and Herpesvirus ateles: human lymphocyte transformation correlates with distinct signaling propertiesof viral oncoproteins. Virus Research 2012.

42. Tybulewicz VL, Henderson RB: Rho family GTPases and their regulators in lymphocytes. Nat Rev Immunol 2009, 9:630-644.

43. Busche S, Descot A, Julien S, Genth H, Posern G: Epithelial cell-cell contacts regulate SRF-mediated transcription via Rac-actin-MAL signalling. J Cell Sci 2008, 121:1025-1035.

44. Brandes V, Schelle I, Brinkmann S, Schulz F, Schwarz J, Gerhard R, Genth H: Protection from $\mathrm{C}$. difficile Toxin B-catalysed $\mathrm{Rac} / \mathrm{Cdc} 42$ glucosylation by tauroursodeoxycholic acid-induced Rac/Cdc42 phosphorylation. Biol Chem 2012, 393:77-84 
45. Halabi-Cabezon I, Huelsenbeck J, May M, Ladwein M, Rottner K, Just I, Genth H: Prevention of the cytopathic effect induced by Clostridium difficile Toxin B by active Rac1. FEBS Lett 2008, 582:3751-3756.

46. Shan X, Czar MJ, Bunnell SC, Liu P, Liu Y, Schwartzberg PL, Wange RL: Deficiency of PTEN in Jurkat T cells causes constitutive localization of Itk to the plasma membrane and hyperresponsiveness to CD3 stimulation. Mol Cell Biol 2000, 20:6945-6957.

47. Descot A, Rex-Haffner M, Courtois G, Bluteau D, Menssen A, Mercher T, Bernard OA, Treisman R, Posern G: OTT-MAL is a deregulated activator of serum response factor-dependent gene expression. Mol Cell Biol 2008, 28:6171-6181.

48. Medjkane S, Perez-Sanchez C, Gaggioli C, Sahai E, Treisman R: Myocardinrelated transcription factors and SRF are required for cytoskeletal dynamics and experimental metastasis. Nat Cell Biol 2009, 11:257-268.

49. Richter S: The influence of Herpesvirus saimiri oncoprotein Tip on apoptosis signaling pathways in T cells. PhD Thesis Friedrich-AlexanderUniversität Erlangen-Nürnberg, Naturwissenschaftliche Fakultät; 2010.

50. Posern G, Sotiropoulos A, Treisman R: Mutant actins demonstrate a role for unpolymerized actin in control of transcription by serum response factor. Mol Biol Cell 2002, 13:4167-4178.

51. Geneste O, Copeland JW, Treisman R: LIM kinase and Diaphanous cooperate to regulate serum response factor and actin dynamics. J Cell Biol 2002, 157:831-838

52. de Jong SJ, Albrecht JC, Schmidt M, Müller-Fleckenstein I, Biesinger B: Activation of noncanonical NF-kappaB signaling by the oncoprotein Tio. J Biol Chem 2010, 285:16495-16503.

doi:10.1186/1478-811X-10-5

Cite this article as: Katsch et al: Actin-dependent activation of serum response factor in T cells by the viral oncoprotein tip. Cell

Communication and Signaling 2012 10:5.

\section{Submit your next manuscript to BioMed Central} and take full advantage of:

- Convenient online submission

- Thorough peer review

- No space constraints or color figure charges

- Immediate publication on acceptance

- Inclusion in PubMed, CAS, Scopus and Google Scholar

- Research which is freely available for redistribution

Submit your manuscript at www.biomedcentral.com/submit
Biomed Central 